\title{
Thermosolutal Magneto - Rotatory Convection In Couple - Stress Fluid through Porous Medium
}

\author{
P. Kumar \\ Associate Professor, Dept. of Mathematics, ICDEOL, Himachal Pradesh University, Shimla-171005, H.P. India. \\ Corresponding Author Email: drpardeep@sancharnet.in; pkdureja@gmail.com
}

(Received August 11, 2010; accepted March 31 ${ }^{\text {th }}, 2011$ )

\begin{abstract}
The thermosolutal convection in a couple-stress fluid layer heated and soluted from below in porous medium is considered to include the effects of uniform vertical magnetic field and uniform vertical rotation. Following the linearized stability theory and normal mode analysis, the dispersion relation is obtained. For the case of stationary convection, the stable solute gradient and rotation have stabilizing effects on the system. In the presence of rotation, the medium permeability has a destabilizing (or stabilizing) effect whereas magnetic field and couple-stress parameter have stabilizing (or destabilizing) effect on the system. On the other hand, in the absence of rotation, medium permeability has a destabilizing effect whereas magnetic field and couple-stress parameter have a stabilizing effect. The dispersion relation is also analyzed numerically. The stable solute gradient, rotation and magnetic field introduce oscillatory modes in the system, which were nonexistent in their absence. A condition for the system to be stable is obtained by using Rayleigh-Ritz inequality.
\end{abstract}

Keywords: Thermosolutal convection, Couple-stress fluid, Porous medium, Uniform vertical rotation, Uniform vertical magnetic field

\section{INTRODUCTION}

The theoretical and experimental results on thermal convection in a fluid layer, in the absence and presence of rotation and magnetic field have been given by Chandrasekhar (1961). Thermal convection is the most convective instability when crystals are produced from single element like silicon. However, gallium arsenide and other semi-conductors which require crystals made from compounds of elements are beginning to take on a prominent position in modern technologies. Hence, at present, there is a strong industrial demand for understanding the additional effects that can occur in the solidification of a mixture, which do not take place in one component system. The problem of thermohaline convection in a layer of fluid heated from below and subjected to a stable salinity gradient has been considered by Veronis (1965). The buoyancy force can arise not only from density differences due to variations in temperature but also from those due to variations in solute concentration. Double-diffusive convection problems arise in oceanography (salt fingers occur in the ocean when hot saline water overlies cooler fresher water which believed to play an important role in the mixing of properties in several regions of the ocean), limnology and engineering. The migration of moisture in fibrous insulation, bio/chemical contaminants transport in environment, underground disposal of nuclear wastes, magmas, groundwater, high quality crystal production and production of pure medication are some examples where double-diffusive convection is involved. Examples of particular interest are provided by ponds built to trap solar heat (Tabor et al. 1965) and some Antarctic lakes (Shirtcliffe, 1967). The physics is quite similar in the stellar case in that helium acts like salt in raising the density and in diffusing more slowly than heat. The conditions under which convective motions are important in stellar atmospheres are usually far removed from consideration of a single component fluid and rigid boundaries, and therefore it is desirable to consider a fluid acted on by a solute gradient and free boundaries.

The flow through porous media is of considerable interest for petroleum engineers, for geophysical fluid dynamicists and has importance in chemical technology and industry. An example in the geophysical context is the recovery of crude oil from the pores of reservoir rocks. Among the applications in engineering disciplines one can find the food processing industry, chemical processing industry, solidification and centrifugal casting of metals. Such flows has shown their great importance in petroleum engineering to study the movement of natural gas, oil and water through the oil reservoirs; in chemical engineering for filtration and purification processes and in the field of agriculture engineering to study the underground water resources, seepage of water in river beds. The problem of thermosolutal convection in fluids in a porous 
medium is of importance in geophysics, soil sciences, ground water hydrology and astrophysics. The study of thermosolutal convection in fluid saturated porous media, has diverse practical applications, including that related to the materials processing technology, in particular, the melting and solidification of binary alloys. The development of geothermal power resources has increased general interest in the properties of convection in porous media. The scientific importance of the field has also increased because hydrothermal circulation is the dominant heat-transfer mechanism in young oceanic crust (Lister, 1972). Generally it is accepted that comets consists of a dusty 'snowball' of a mixture of frozen gases which in the process of their journey changes from solid to gas and vice - versa. The physical properties of comets, meteorites and interplanetary dust strongly suggest the importance of porosity in the astrophysical context (McDonnel, 1978). The effect of a magnetic field on the stability of such a flow is of interest in geophysics, particularly in the study of Earth's core where the Earth's mantle, which consists of conducting fluid, behaves like a porous medium which can become convectively unstable as a result of differential diffusion. The other application of the results of flow through a porous medium in the presence of a magnetic field is in the study of the stability of a convective flow in the geothermal region. Also the magnetic field in double-diffusive convection has its importance in the fields of engineering, for example, MHD generators and astrophysics particularly in explaining the properties of large stars with a helium rich core. Stommel et al. (1967) and Linden (1974) have remarked that the length scales characteristics of double-diffusive convective layers in the ocean may be sufficiently large that the Earth's rotation might be important in their formation. Moreover, the rotation of the Earth distorts the boundaries of a hexagonal convection cell in a fluid through a porous medium and the distortion plays an important role in the extraction of energy in the geothermal regions. Brakke (1955) explained a double - diffusive instability that occurs when a solution of a slowly diffusing protein is layered over a denser solution of more rapidly diffusing sucrose. Nason et al. (1969) found that this instability, which is deleterious to certain biochemical separations, can be suppressed by rotation in the ultracentrifuge.

The theory of couple-stress fluid has been formulated by Stokes (1966). One of the applications of couplestress fluid is its use to the study of the mechanisms of lubrications of synovial joints, which has become the object of scientific research. A human joint is a dynamically loaded bearing which has articular cartilage as the bearing and synovial fluid as the lubricant. When a fluid film is generated, squeeze - film action is capable of providing considerable protection to the cartilage surface. The shoulder, ankle, knee and hip joints are the loaded - bearing synovial joints of the human body and these joints have a low friction coefficient and negligible wear. Normal synovial fluid is a viscous, non-Newtonian fluid and is clear or yellowish. According to the theory of Stokes (1966), couple-stresses appear in noticeable magnitudes in fluids with very large molecules. Since the long chain hyaluronic acid molecules are found as additives in synovial fluids, Walicki et al. (1999) modeled the synovial fluid as a couple-stress fluid. The synovial fluid is the natural lubricant of joints of the vertebrates. The detailed description of the joint lubrication has very important practical implications. Practically all diseases of joints are caused by or connected with malfunction of the lubrication. The efficiency of the physiological joint lubrication is caused by several mechanisms. The synovial fluid is due to its content of the hyaluronic acid, a fluid of high viscosity, near to gel. Goel et al. (1999) have studied the hydromagnetic stability of an unbounded couple-stress binary fluid mixture under rotation with vertical temperature and concentration gradients. Sharma et al. (2002) have considered a couple - stress fluid with suspended particles heated from below. In another study, Sunil et al. (2002) have considered a couple- stress fluid heated from below in a porous medium in the presence of a magnetic field and rotation. Kumar et al. (2004) have considered the thermal instability of a layer of couple-stress fluid acted on by a uniform rotation, and have found that for stationary convection the rotation has a stabilizing effect whereas couple-stress has both stabilizing and destabilizing effects.

Keeping in mind the importance in geophysics, soil sciences, ground water hydrology, astrophysics and various applications mentioned above, the thermosolutal convection in couple-stress fluid in porous medium in the presence of uniform rotation and uniform magnetic field has been considered in the present paper.

\section{Formulation of the Problem AND Perturbation Equations}

Here we consider an infinite, horizontal, incompressible couple-stress fluid layer of thickness $d$, heated and soluted from below so that, the temperatures, densities and solute concentrations at the bottom surface $\mathrm{z}=0$ are $\mathrm{T}_{0}, \rho_{0}$ and $\mathrm{C}_{0}$ and at the upper surface $\mathrm{z}=\mathrm{d}$ are $\mathrm{T}_{\mathrm{d}}$, $\rho_{\mathrm{d}}$ and $\mathrm{C}_{\mathrm{d}}$ respectively, and that a uniform temperature gradient $\beta\left(=\left|\frac{d T}{d z}\right|\right)$ and a uniform solute gradient $\beta^{\prime}\left(=\left|\frac{d C}{d z}\right|\right)$ are maintained. The gravity field $\vec{g}(0,0,-g)$, a uniform vertical magnetic field $\vec{H}(0,0, H)$ and a uniform vertical rotation $\vec{\Omega}(0,0, \Omega)$ pervade the system. This fluid layer is assumed to be flowing through an isotropic and homogeneous porous medium of porosity $\in$ and medium permeability $\mathrm{k}_{1}$.

Let $\mathrm{p}, \rho, \mathrm{T}, \mathrm{C}, \alpha, \alpha^{\prime}, \mathrm{g}, \eta, \mu_{\mathrm{e}}$ and $\vec{q}(u, v, w)$ denote respectively, the fluid pressure, density, temperature, solute concentration, thermal coefficient of expansion, an analogous solvent coefficient of expansion, gravitational acceleration, resistivity, magnetic permeability and fluid velocity. The equations expressing the conservation of momentum, mass, temperature, solute concentration and equation of state of couple-stress fluid are: 


$$
\begin{aligned}
& \frac{1}{\varepsilon}\left[\frac{\partial \vec{q}}{\partial t}+\frac{1}{\varepsilon}(\vec{q} \cdot \nabla) \vec{q}\right]= \\
& -\left(\frac{1}{\rho_{0}}\right) \nabla p+\vec{g}\left(1+\frac{\delta \rho}{\rho_{0}}\right)-\frac{1}{k_{1}}\left(v-\frac{\mu^{\prime}}{\rho_{0}} \nabla^{2}\right) \vec{q} \\
& +\frac{\mu_{e}}{4 \pi \rho_{0}}(\nabla \times \vec{H}) \times \vec{H}+\frac{2}{\varepsilon}(\vec{q} \times \vec{\Omega}) \\
& \nabla \cdot \vec{q}=0 \\
& E \frac{\partial T}{\partial t}+(\vec{q} \cdot \nabla) T=\chi \nabla^{2} T \\
& E^{\prime} \frac{\partial C}{\partial t}+(\vec{q} \cdot \nabla) C=\chi^{\prime} \nabla^{2} C \\
& \rho=\rho_{0}\left[1-\alpha\left(T-T_{0}\right)+\alpha^{\prime}\left(C-C_{0}\right)\right.
\end{aligned}
$$

where the suffix zero refers to values at the reference level $z=0$ and in writing Eq. (1), use has been made of Boussinesq approximation. The magnetic permeability $\mu_{\mathrm{e}}$, the kinematic viscosity $\nu$, couple-stress viscosity $\mu^{\prime}$, the thermal diffusivity $\chi$ and the solute diffusivity $\chi^{\prime}$ are all assumed to be constants. The Maxwell's equations yield

$\varepsilon \frac{d \vec{H}}{d t}=(\vec{H} \cdot \nabla) \vec{q}+\varepsilon \eta \nabla^{2} \vec{H}$

and

$$
\nabla \cdot \vec{H}=0
$$

where $\frac{d}{d t} \equiv \frac{\partial}{\partial t}+\varepsilon^{-1} \vec{q} \cdot \nabla$ stands for the convective derivative. Here $E=\varepsilon+(1-\varepsilon)\left(\frac{\rho_{s} C_{s}}{\rho_{0} C_{i}}\right)$ is a constant and $\mathrm{E}^{\prime}$ is a constant analogous to $\mathrm{E}$ but corresponding to solute rather that heat; $\rho_{s}, C_{s}$ and $\rho_{o}, C_{i}$ stand for density and heat capacity of solid (porous matrix) material and fluid, respectively. The steady state solution is

$$
\begin{aligned}
& \vec{q}=(0,0,0), T=T_{0}-\beta z, C=C_{0}-\beta / z \\
& \rho=\rho_{0}\left(1+\alpha \beta z+\alpha^{\prime} \beta^{\prime} z\right)
\end{aligned}
$$

Here we use linearized stability theory and normal mode analysis method. Consider a small perturbation on the steady state solution, and let $\delta p, \delta \rho, \theta, \gamma$, $\vec{h}\left(h_{x}, h_{y}, h_{z}\right)$ and $\vec{q}(\mathrm{u}, \mathrm{v}, \mathrm{w})$ denote, respectively, the perturbations in pressure $\mathrm{p}$, density $\rho$, temperature $\mathrm{T}$, solute concentration $\mathrm{C}$, magnetic field $\vec{H}(0,0,0)$ and velocity $\vec{q}(0,0,0)$. The change in density $\delta \rho$, caused mainly by the perturbations $\theta$ and $\gamma$ in temperature and concentration, is given by

$$
\delta \rho=-\rho_{0}\left(\alpha \theta-\alpha^{\prime} \gamma\right)
$$

Then the linearized perturbation equations become

$$
\begin{aligned}
& \frac{1}{\varepsilon} \frac{\partial \vec{q}}{\partial t}=-\frac{1}{\rho_{0}} \nabla \delta p-\vec{g}\left(\alpha \theta-\alpha^{\prime} \gamma\right)-\frac{1}{k_{1}}\left(v-\frac{\mu^{\prime}}{\rho_{0}} \nabla^{2}\right) \overrightarrow{q+} \\
& \frac{\mu_{e}}{4 \pi \rho_{0}}(\nabla \times \vec{h}) \times \vec{H}+\frac{2}{\varepsilon}(\vec{q} \times \vec{\Omega})
\end{aligned}
$$

$\nabla \cdot \vec{q}=0$

$E \frac{\partial \theta}{\partial t}=\beta w+\chi \nabla^{2} \theta$

$E^{\prime} \frac{\partial \gamma}{\partial t}=\beta^{\prime} w+\chi^{\prime} \nabla^{2} \gamma$

$\varepsilon \frac{\partial \vec{h}}{\partial t}(\vec{H} \cdot \nabla) \vec{q}+\varepsilon \eta \nabla^{2} \vec{h}$

and

$\nabla \cdot \vec{h}=0$

\section{THE DISPERSION RELATION}

Analyzing the disturbances into normal modes, we assume that the perturbation quantities are of the form

$\left[\mathrm{w}, \mathrm{h}_{\mathrm{z}}, \theta, \gamma, \zeta, \xi\right]=[\mathrm{W}(\mathrm{z}), \quad \mathrm{K}(\mathrm{z}), \quad \Theta(\mathrm{z}), \quad \Gamma(\mathrm{z})$, $\mathrm{Z}(\mathrm{z}), \mathrm{X}(\mathrm{z})] \exp \left(\mathrm{ik}_{\mathrm{x}} \mathrm{x}+\mathrm{ik}_{\mathrm{y}} \mathrm{y}+\mathrm{nt}\right)$

where $\mathrm{k}_{\mathrm{x}}, \mathrm{k}_{\mathrm{y}}$ are the wave numbers along the $\mathrm{x}$ - and $\mathrm{y}$ directions respectively, $\mathrm{k}=\left(\sqrt{{k_{x}^{2}+k_{y}^{2}}^{2}}\right)$ is the resultant wave number and $\mathrm{n}$ is the growth rate which is, in general, a complex constant. $\xi=\frac{\partial v}{\partial x}-\frac{\partial u}{\partial y} \quad$ and $\xi=\frac{\partial h_{y}}{\partial x}-\frac{\partial h_{x}}{\partial y}$ stand for the z-components of vorticity and current density, respectively.

Expressing the coordinates $\mathrm{x}, \mathrm{y}, \mathrm{z}$ in the new unit of length $\mathrm{d}$ and letting $\mathrm{a}=\mathrm{kd}, \sigma=\frac{n d^{2}}{v}, p_{1}=\frac{v}{\chi}$ (Prandtl number), $p_{2}=\frac{v}{\eta}$ (magnetic Prandtl number), $q=\frac{v}{\chi^{\prime}}$ (Schmidt number), $P_{l}=\frac{k_{1}}{d^{2}}$ dimensionless medium permeability) and $\mathrm{F}=\frac{\mu^{\prime}}{\rho_{0} d^{2} v}$ (dimensionless couplestress parameter), Eq. (10) to Eq. (15), using Eq. (16), yield

$$
\begin{aligned}
& {\left[\frac{\sigma}{\varepsilon}+\frac{1}{P_{l}}-\frac{F}{P_{l}}\left(D^{2}-a^{2}\right)\right]\left(D^{2}-a^{2}\right) W+\frac{g a^{2} d^{2}}{v}(\alpha \Theta-\alpha \Gamma)} \\
& -\frac{\mu_{e} H d}{4 \pi \rho_{0} v}\left(D^{2}-a^{2}\right) D K+\frac{2 \Omega d^{3}}{\varepsilon v} D Z=0
\end{aligned}
$$

$\left[\frac{\sigma}{\varepsilon}+\frac{1}{P_{l}}-\frac{F}{P_{l}}\left(D^{2}-a^{2}\right)\right] Z$

$=\left(\frac{\mu_{e} H d}{4 \pi \rho_{0} v}\right) D X+\left(\frac{2 \Omega d}{\varepsilon v}\right) D W$

$\left(D^{2}-a^{2}-p_{2} \sigma\right) K=-\left(\frac{H d}{\varepsilon \eta}\right) D W$

$\left(D^{2}-a^{2}-p_{2} \sigma\right) X=-\left(\frac{H d}{\varepsilon \eta}\right) D Z$

$\left(D^{2}-a^{2}-E p_{1} \sigma\right) \Theta=-\left(\frac{\beta d^{2}}{\chi}\right) W$

$\left(D^{2}-a^{2}-E^{\prime} q \sigma\right) \Gamma=-\left(\frac{\beta^{\prime} d^{2}}{\chi^{\prime}}\right) W$ 
Consider the case where both boundaries are free as well as perfect conductors of both heat and solute concentration, while the adjoining medium is perfectly conducting. The case of two free boundaries is a little artificial but it enables us to find analytical solutions and to make some qualitative conclusions. The appropriate boundary conditions, with respect to which Eqs. (17) - (22) must be solved are

$$
W=D^{2} W=X=D Z=0, \Theta=0, \Gamma=0 \text { at } \mathrm{z}=0
$$

and $\mathrm{z}=1$

$\mathrm{DX}=0, \mathrm{~K}=0$ on a perfectly conducting boundary and $\mathrm{X}=0$ and $\mathrm{h}_{\mathrm{x}}, \mathrm{h}_{\mathrm{y}}, \mathrm{h}_{\mathrm{z}}$ are continuous with an external vacuum field on a non-conducting boundary.

The case of two free boundaries, though little artificial, is the most appropriate for stellar atmospheres (Spiegel, 1965). Using the above boundary conditions, it can be shown that all the even order derivatives of $\mathrm{W}$ must vanish for $\mathrm{z}=0$ and $\mathrm{z}=1$ and hence the proper solution of $\mathrm{W}$ charactering the lowest mode is

$W=W_{0} \sin \pi z$

where $\mathrm{W}_{0}$ is a constant.

Eliminating $\Theta, \Gamma, \mathrm{K}, \mathrm{Z}$ and $\mathrm{X}$ between Eqs. (17) - (22) and substituting the proper solution $\mathrm{W}=\mathrm{W}_{0} \sin \pi \mathrm{z}$, in the resultant equation, we obtain the dispersion relation

$$
\begin{aligned}
& R_{1}=\left(\frac{1+x}{x}\right)\left(1+x+i E p_{1} \sigma_{1}\right) \\
& {\left[\begin{array}{l}
\left\{\frac{i \sigma_{1}}{\in}+\frac{1}{P}+\frac{F_{1}}{P}(1+x)\right\} \\
\left(1+x+i p_{2} \sigma_{1}\right)+Q_{1}
\end{array}\right]} \\
& +S_{1} \frac{\left(1+x+i E p_{1} \sigma_{1}\right)}{\left(1+x+i E^{\prime} q \sigma_{1}\right)}+ \\
& T_{1} \frac{\left[\begin{array}{l}
\left(1+x+i E p_{1} \sigma_{1}\right) \\
\left(1+x+i p_{2} \sigma_{1}\right)
\end{array}\right]}{\left[\left\{\begin{array}{l}
\left\{\frac{i \sigma_{1}}{\epsilon}+\frac{1}{P}+\frac{F_{1}}{P}(1+x)\right\} \\
\left(1+x+i p_{2} \sigma_{1}\right)+Q_{1}
\end{array}\right]\right.} \\
& T_{1}=\left(\frac{2 \Omega d^{2}}{\varepsilon v \pi^{2}}\right)^{2}, x=\frac{a^{2}}{\pi^{2}}, \quad i \sigma_{1}=\frac{\sigma}{\pi^{2}} \quad, \quad F=\pi^{2} F \text { and } \\
& P=\pi^{2} P
\end{aligned}
$$

\section{The Stationary CONVECTION}

When the instability sets in as stationary convection, the marginal state will be characterized by $\sigma=0$. Putting $\sigma=0$, the dispersion relation (25) reduces to

$$
\begin{aligned}
& R_{1}=\frac{(1+x)^{2}}{x}\left[\left\{\frac{1}{P}+\frac{F_{1}}{P}(1+x)\right\}(1+x)+Q_{1}\right] \\
& +S_{1}+T_{1} \frac{(1+x)^{2}}{x\left[\left\{\frac{1}{P}+\frac{F_{1}}{P}(1+x)\right\}(1+x)+Q_{1}\right]}
\end{aligned}
$$

To investigate the effects of stable solute gradient, rotation, magnetic field, couple-stress parameter and medium permeability, we examine the behaviour of $\frac{d R_{1}}{d S_{1}}, \frac{d R_{1}}{d T_{1}}, \frac{d R_{1}}{d Q_{1}}, \frac{d R_{1}}{d F_{1}}$ and $\frac{d R_{1}}{d P}$ analytically.

Equation (26) yields:

$$
\begin{aligned}
& \frac{d R_{1}}{d S_{1}}=+1 \\
& \frac{d R_{1}}{d S_{1}}=\frac{(1+x)^{2}}{x\left[\left\{\frac{1}{P}+\frac{F_{1}}{P}(1+x)\right\}(1+x)+Q_{1}\right]} \\
& \frac{d R_{1}}{d Q_{1}}=\frac{(1+x)^{2}}{x}\left[1-\frac{T_{1}}{\left[\left\{\frac{1}{P}+\frac{F_{1}}{P}(1+x)\right\}(1+x)+Q_{1}\right]^{2}}\right]
\end{aligned}
$$

$$
\frac{d R_{1}}{d F_{1}}=\frac{(1+x)^{4}}{x P}\left[1-\frac{T_{1}}{\left[\left\{\frac{1}{P}+\frac{F_{1}}{P}(1+x)\right\}(1+x)+Q_{1}\right]^{2}}\right]
$$

$$
\frac{d R_{1}}{d P}=-\frac{(1+x)^{3}}{x P^{2}}\left[1-\frac{T_{1}}{\left[\left\{\frac{1}{P}+\frac{F_{1}}{P}(1+x)\right\}(1+x)+Q_{1}\right]^{2}}\right]
$$

Equations (27) and (28) imply that, for stationary convection, stable solute gradient and rotation have stabilizing effects on the system. Equations (29) - (31) shows that in the absence of rotation $\left(\mathrm{T}_{1}=0\right), \frac{d R_{1}}{d Q_{1}}$ and $\frac{d R_{1}}{d F_{1}}$ is always positive whereas $\frac{d R_{1}}{d P_{1}}$ is always

negative which means that magnetic field and couplestress parameter have a stabilizing effect, whereas, medium permeability has a destabilizing effect on the system in the absence of rotation. For a rotating system, the magnetic field and couple-stress parameter have a stabilizing ( or destabilizing) effect and the medium permeability has a destabilizing ( or stabilizing) effect on instability of couple- stress rotating fluid in porous medium in hydromagnetics if

$$
T_{1} \prec(\text { or } \succ)\left[\left\{\frac{1}{P}+\frac{F_{1}}{P}(1+x)\right\}(1+x)+Q_{1}\right]^{2}
$$

The dispersion relation (26) is also analyzed numerically for various values of $\mathrm{S}_{1}, \mathrm{~T}_{1}, \mathrm{P}, \mathrm{F}_{1}$ and $\mathrm{Q}_{1}$. It 
is also evident from Figs. 1 - 5 that stable solute gradient and rotation have stabilizing effects whereas magnetic field, couple-stress parameter and medium permeability have both stabilizing and destabilizing effects on the system.

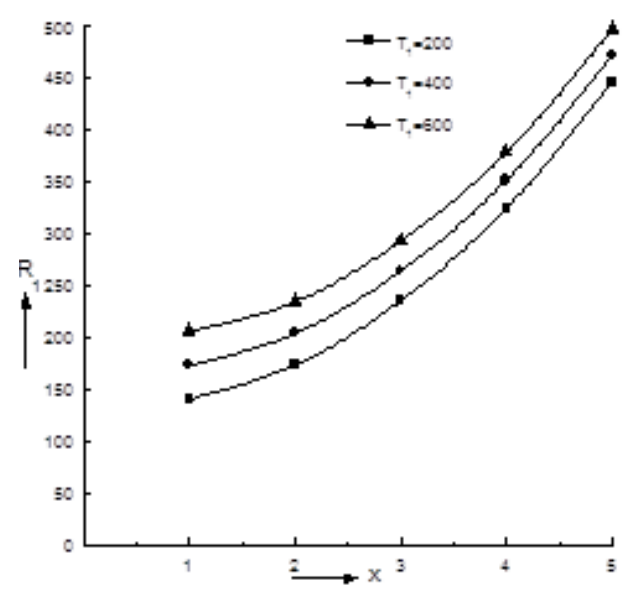

Fig.1. The variation of Rayleigh number $R_{1}$ with $\mathrm{x}$ for $S_{1}=10, F_{1}=5, Q_{1}=20, \mathrm{P}=5, T_{1}=200,400$ and 600 .

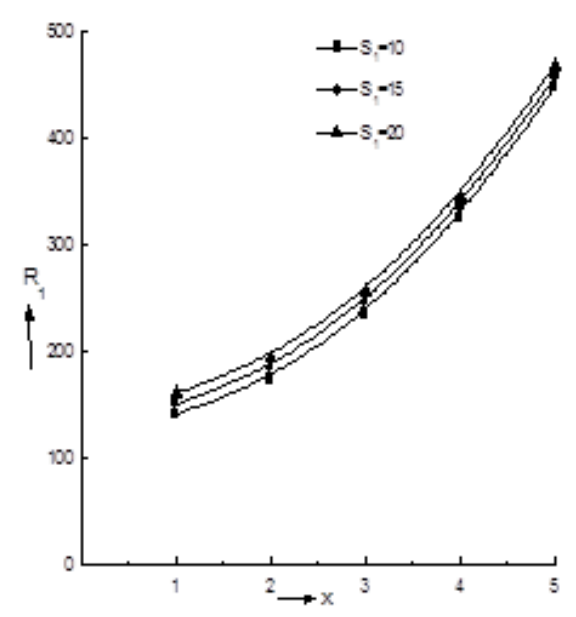

Fig.2. The variation of Rayleigh number $R_{1}$ with $\mathrm{x}$ for, $F_{1}=5, Q_{1}=20, \mathrm{P}=5, T_{1}=200, S_{1}=10,15$ and 20.

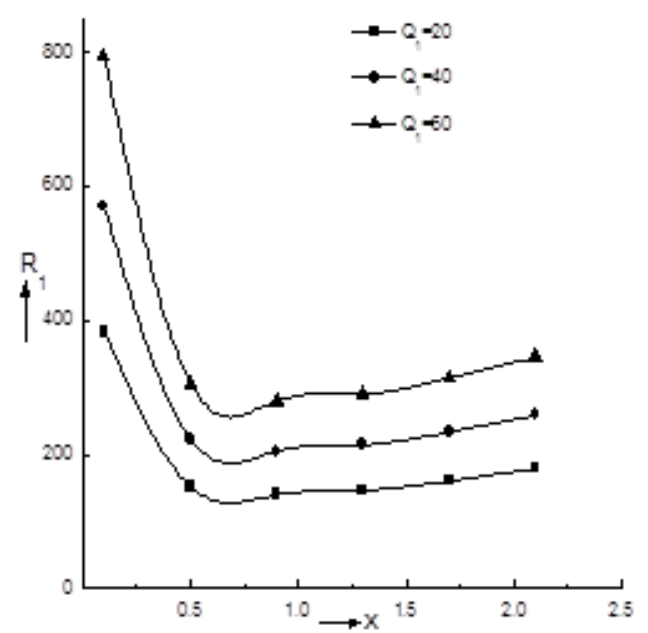

Fig.3. The variation of Rayleigh number $R_{1}$ with $\mathrm{x}$ for $S_{1}=10, F_{1}=5, \mathrm{P}=5, T_{1}=200, Q_{1}=20,40$ and 60.

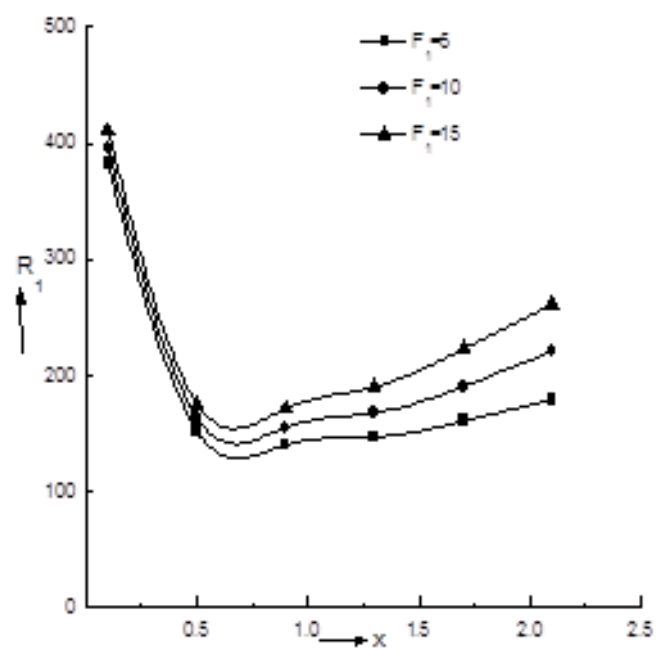

Fig.4. The variation of Rayleigh number $R_{1}$ with $\mathrm{x}$ for $S_{1}=10, Q_{1}=20, \mathrm{P}=5, T_{1}=200, F_{1}=5,10$ and 15.

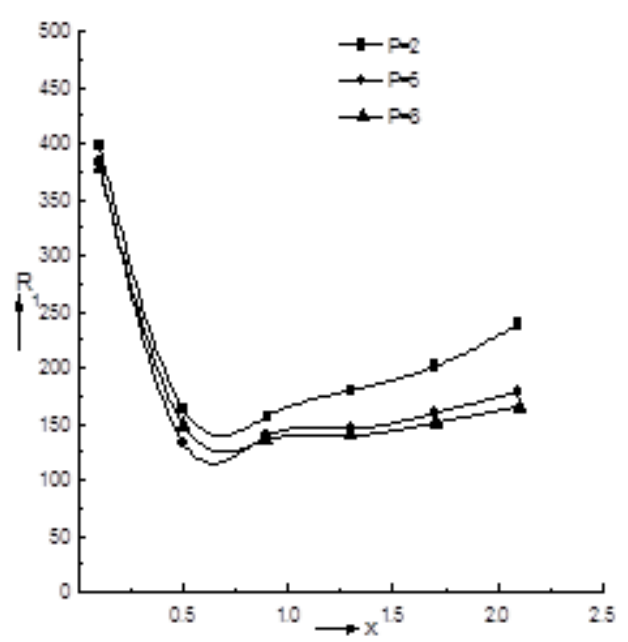

Fig.5. The variation of Rayleigh number $R_{1}$ with $\mathrm{x}$ for $S_{1}=10, F_{1}=5, Q_{1}=20, T_{1}=200, \mathrm{P}=2,5$ and 8.

\section{SOME IMPORTANT THEOREMS}

Theorem 1: The system is stable or unstable.

Proof: Multiplying Eq. (17) by $\mathrm{W}^{*}$, the complex conjugate of $\mathrm{W}$, integrating over the range of $\mathrm{z}$ and making use of Eqs. (18) - (22) together with the boundary conditions (23), we obtain

$$
\begin{aligned}
& -\left[\frac{\sigma}{\varepsilon}+\frac{1}{P_{l}}\right] I_{1}-\frac{F}{P_{l}} I_{2}+\frac{g a^{2} \alpha \chi}{\beta v}\left[I_{3}+E p_{1} \sigma^{*} I_{4}\right] \\
& -\frac{g a^{2} \alpha^{\prime} \chi^{\prime}}{\beta^{\prime} v}\left[I_{5}+E^{\prime} q \sigma^{*} I_{6}\right]-\frac{\mu_{e} \varepsilon \eta}{4 \pi \rho_{0} v}\left[I_{7}+p_{2} \sigma^{*} I_{8}\right] \\
& -\frac{d^{2} F}{P_{l}} I_{9}-d^{2}\left[\frac{\sigma^{*}}{\varepsilon}+\frac{1}{P_{l}}\right] I_{10} \\
& -\frac{\mu_{e} d^{2} \varepsilon \eta}{4 \pi \rho_{0} v} I_{11}-\frac{\mu_{e} d^{2} \varepsilon \eta p_{2} \sigma}{4 \pi \rho_{0} v} I_{12}=0
\end{aligned}
$$

where 


$$
\begin{aligned}
& I_{1}=\int_{0}^{1}\left(|D W|^{2}+a^{2}|W|^{2}\right) d z \\
& I_{2}=\int_{0}^{1}\left(\left|D^{2} W\right|^{2}+2 a^{2}|D W|^{2}+a^{4}|W|^{2}\right) d z \\
& I_{3}=\int_{0}^{1}\left(|D \Theta|^{2}+a^{2}|\Theta|^{2}\right) d z ; I_{4}=\int_{0}^{1}\left(|\Theta|^{2}\right) d z \\
& I_{5}=\int_{0}^{1}\left(|D \Gamma|^{2}+a^{2}|\Gamma|^{2}\right) d z ; \quad I_{4}=\int_{0}^{1}\left(|\Gamma|^{2}\right) d z \\
& I_{7}=\int_{0}^{1}\left(\left|D^{2} K\right|^{2}+2 a^{2}|D K|^{2}+a^{4}|K|^{2}\right) d z \\
& I_{8}=\int_{0}^{1}\left(|D K|^{2}+a^{2}|K|^{2}\right) d z \\
& I_{9}=\int_{0}^{1}\left(|D Z|^{2}+a^{2}|Z|^{2}\right) d z ; I_{10}=\int_{0}^{1}\left(|Z|^{2}\right) d z \\
& I_{11}=\int_{0}^{1}\left(|D X|^{2}+a^{2}|X|^{2}\right) d z ; I_{12}=\int_{0}^{1}\left(|X|^{2}\right) d z
\end{aligned}
$$

and $\sigma^{*}$ is the complex conjugate of $\sigma$. The integrals $I_{1}-$ $\mathrm{I}_{12}$ are all positive definite. Putting $\sigma=\sigma_{\mathrm{r}}+\mathrm{i} \sigma_{\mathrm{i}}$ and equating the real and imaginary parts of Eq. (33), we obtain

$$
\begin{aligned}
& {\left[\begin{array}{c}
-\frac{I_{1}}{\varepsilon}+\frac{g a^{2} \alpha \chi}{\beta v} E p_{1} I_{4}-\frac{g a^{2} \alpha^{\prime} \chi^{\prime}}{\beta^{\prime} v} E^{\prime} q I_{6}- \\
\frac{\mu_{e} \varepsilon \eta}{4 \pi \rho_{0} v} p_{2} I_{8}-\frac{d^{2}}{\varepsilon} I_{10}+\frac{\mu_{e} d^{2} \varepsilon \eta}{4 \pi \rho_{0} v} p_{2} I_{12}
\end{array}\right] \sigma_{r}=} \\
& \frac{I_{1}}{P_{l}}+\frac{F}{P_{l}} I_{2}-\frac{g a^{2} \alpha \chi}{\beta v} I_{3}+\frac{g a^{2} \alpha^{\prime} \chi^{\prime}}{\beta^{\prime} v} I_{5}+\frac{\mu_{e} \varepsilon \eta}{4 \pi \rho_{0} v} I_{7} \\
& +\frac{d^{2} F}{P_{l}} I_{9}+\frac{d^{2}}{P_{l}} I_{10}+\frac{\mu_{e} d^{2} \varepsilon \eta}{4 \pi \rho_{0} v} I_{11}
\end{aligned}
$$

and

$$
\left[\begin{array}{l}
\frac{I_{1}}{\varepsilon}+\frac{g a^{2} \alpha \chi}{\beta v} E p_{1} I_{4}-\frac{g a^{2} \alpha^{\prime} \chi^{\prime}}{\beta^{\prime} v} E^{\prime} q I_{6}- \\
\frac{\mu_{e} \varepsilon \eta}{4 \pi \rho_{0} v} p_{2} I_{8}-\frac{d^{2}}{\varepsilon} I_{10}+\frac{\mu_{e} d^{2} \varepsilon \eta}{4 \pi \rho_{0} v} p_{2} I_{12}
\end{array}\right] \sigma_{i}=0
$$

It is evident from Eq. (35) that $\sigma_{\mathrm{r}}$ is positive or negative. The system is, therefore, stable or unstable.

Theorem 2: The modes may be oscillatory or nonoscillatory in contrast to case of no magnetic field and no rotation, and in the absence of stable solute gradient where modes are non-oscillatory.

Proof: Eq. (36) yields that $\sigma_{\mathrm{i}}$ may be zero or non-zero, which means that the modes may be non-oscillatory or oscillatory. In the absence of stable solute gradient, rotation and magnetic field, Eq. (36) reduces to

$$
\sigma_{i}\left(\frac{I_{1}}{\varepsilon}+\frac{g a^{2} \alpha \chi}{v \beta} E p_{1} I_{4}\right)=0
$$

and the terms in brackets are positive definite. Thus $\sigma_{\mathrm{i}}$ $=0$, which means that oscillatory modes are not allowed and the principle of exchange of stabilities is valid for the couple-stress fluid for a porous medium, in the absence of stable solute gradient, rotation and magnetic field. The oscillatory modes are introduced due to the presence of stable solute gradient, rotation and magnetic field, which were non-existent in their absence.

Theorem 3: The system is stable for $\frac{g \alpha \chi}{v \beta} \frac{P_{1}}{F} \leq \frac{27 \pi^{4}}{4}$ and under the condition $\frac{g \alpha \chi}{v \beta} \frac{P_{1}}{F} \succ \frac{27 \pi^{4}}{4}$, the system becomes unstable.

Proof: From Eq. (36), it is clear that $\sigma_{i}$ is zero when the quantity multiplying it is not zero and arbitrary when this quantity is zero.

If $\sigma_{\mathrm{i}} \neq 0$, then Eq. (36) gives

$$
\begin{aligned}
& -\frac{I_{1}}{\varepsilon}-\frac{\mu_{e} d^{2} \varepsilon \eta}{4 \pi \rho_{0} v} p_{2} I_{12}=\frac{g a^{2} \alpha \chi}{\beta v} E p_{1} I_{4}- \\
& \frac{g a^{2} \alpha^{\prime} \chi^{\prime}}{\beta^{\prime} v} E^{\prime} q I_{6}-\frac{\mu_{e} \varepsilon \eta}{4 \pi \rho_{0} v} p_{2} I_{8}-\frac{d^{2}}{\varepsilon} I_{10}
\end{aligned}
$$

Substituting in Eq. (35), we have

$$
\begin{aligned}
& \frac{2 \sigma_{r} I_{1}}{\varepsilon}+\frac{1}{P_{l}} I_{1}+\frac{F}{P_{l}} I_{2}+\frac{g a^{2} \alpha^{\prime} \chi^{\prime}}{\beta^{\prime} v} I_{5}+\frac{\mu_{e} \varepsilon \eta}{4 \pi \rho_{0} v} I_{7} \\
& +\frac{d^{2} F}{P_{l}} I_{9}+\frac{d^{2}}{P_{l}} I_{10}+\frac{\mu_{e} d^{2} \varepsilon \eta}{4 \pi \rho_{0} v} I_{11}+\frac{\sigma_{r} \mu_{e} d^{2} \varepsilon \eta}{2 \pi \rho_{0} v} p_{2} I_{12} \\
& =\frac{g a^{2} \alpha \chi}{\beta v} I_{3}
\end{aligned}
$$

Equation (39) on using Rayleigh-Ritz inequality gives

$$
\begin{aligned}
& \frac{\left(\pi^{2}+a^{2}\right)^{3}}{a^{2}} \int_{0}^{1}|W|^{2} d z+\frac{\left(\pi^{2}+a^{2}\right)}{a^{2}} \frac{P_{l}}{F} \\
& \left\{\begin{array}{l}
\frac{\sigma_{r} \mu_{e} d^{2} \varepsilon \eta}{2 \pi \rho_{0} v} p_{2} I_{12}+\frac{\mu_{e} d^{2} \varepsilon \eta}{4 \pi \rho_{0} v} I_{11}+\frac{d^{2}}{P_{l}} I_{10} \\
+\frac{d^{2} F}{P_{l}} I_{9}+\frac{\mu_{e} \varepsilon \eta}{4 \pi \rho_{0} v} I_{7}+\frac{g a^{2} \alpha^{\prime} \chi^{\prime}}{\beta^{\prime} v} I_{5}+\frac{1}{P_{l}} I_{1}+\frac{2 \sigma_{r}}{\varepsilon} I_{1}
\end{array}\right\} \\
& \leq \frac{g \alpha \chi}{\beta v} \frac{P_{l}}{F} \int_{0}^{1}|W|^{2} d z
\end{aligned}
$$

Therefore, it follows from Eq. (40) that

$$
\begin{aligned}
& {\left[\frac{27 \pi^{4}}{4}-\frac{g \alpha \chi}{v \beta} \frac{P_{l}}{F}\right] \int_{0}^{1}|W|^{2} d z+\frac{\left(\pi^{2}+a^{2}\right)}{a^{2}} \frac{P_{l}}{F}} \\
& \left\{\begin{array}{l}
\frac{\sigma_{r} \mu_{e} d^{2} \in \eta}{2 \pi \rho_{0} v} p_{2} I_{12}+\frac{\mu_{e} d^{2} \in \eta}{4 \pi \rho_{0} v} I_{11}+\frac{d^{2}}{P_{l}} I_{10} \\
+\frac{d^{2} F}{P_{l}} I_{9}+\frac{\mu_{e} \in \eta}{4 \pi \rho_{0} v} I_{7}+\frac{g a^{2} \alpha^{\prime} \chi^{\prime}}{\beta^{\prime} v} I_{5}+
\end{array}\right.
\end{aligned}
$$




$$
\left.\frac{1}{P_{l}} I_{1}+\frac{2 \sigma_{r} I_{1}}{\in}\right\} \leq 0
$$

since minimum value of $\frac{\left(\pi^{2}+a^{2}\right)^{3}}{a^{2}}$ with respect to $a^{2}$ is $\frac{27 \pi^{4}}{4}$.

Now, let $\sigma_{\mathrm{r}} \geq 0$, we necessary have from (41) that

$$
\frac{g \alpha \chi}{v \beta} \frac{P_{1}}{F} \succ \frac{27 \pi^{4}}{4}
$$

Hence, if

$$
\frac{g \alpha \chi}{v \beta} \frac{P_{1}}{F} \leq \frac{27 \pi^{4}}{4}
$$

then $\sigma_{\mathrm{r}}<0$. Therefore, the system is stable. Therefore, under condition (43), the system is stable and under condition (42) the system becomes unstable.

\section{Conclusions}

The effect of a uniform vertical magnetic field and uniform rotation on the thermosolutal convection in a couple-stress fluid heated and soluted from below in porous medium is considered in the present paper. The investigation of thermosolutal convection is motivated by its interesting complexities as a double diffusion phenomena as well as its direct relevance to geophysics and astrophysics. The thermosolutal convection problems arise in oceanography, limnology and engineering. Ponds built to trap solar heat and some Antartic lakes provide examples of particular interest. The main conclusions from the analysis of this paper are as follows:

a) For the case of stationary convection the following observations are made:

- The stable solute gradient and rotation have stabilizing effect on the system.

- In the presence of rotation, the medium permeability has a destabilizing (or stabilizing) effect whereas magnetic field and couple-stress parameter have stabilizing (or destabilizing) effect on the system.

- In the absence of rotation, the medium permeability has a destabilizing effect whereas magnetic field and couple-stress parameter have a stabilizing effect.

b) It is also observed from Figs. 1-5 that stable solute gradient and rotation have stabilizing effects whereas magnetic field, couple - stress parameter and medium permeability have both stabilizing and destabilizing effects on the system.

c) It is observed that stable solute gradient, rotation and magnetic field introduce oscillatory modes in the system, which was non-existent in their absence. d) In the absence of stable solute gradient, rotation and magnetic field, oscillatory modes are not allowed and the principle of exchange of stabilities is valid.

e) It is found that if $\frac{g \alpha \chi}{v \beta} \frac{P_{1}}{F} \leq \frac{27 \pi^{4}}{4}$ the system is stable and under the condition $\frac{g \alpha \chi}{v \beta} \frac{P_{1}}{F} \succ \frac{27 \pi^{4}}{4}, \quad$ the $\quad$ system becomes unstable.

\section{ACKNOWLEDGEMENTS}

The author is grateful to the learned referee for his critical comments, which led to a significant improvement of the paper.

\section{REFERENCES}

Brakke, M.K. (1955). Zone electrophoresis of dyes, proteins and viruses in density- gradient columns of sucrose solutions, Arch. Biochem. Biophys., Vol. 55, 175-190

Chandresekhar, S. (1961). Hydrodynamic and Hydromagnetic Stability, Clarendon Press, Oxford, UK.

Goel, A.K., S.C. Agarwal and G.S. Agarwal (1999). Hydromagnetic stability of an unbounded couple-stress binary fluid mixture having vertical temperature and concentration gradients with rotation, Indian J. Pure Appl. Math., Vol. 30, 991-1001.

Kumar, P., R. Lal and P. Sharma (2004). Effect of rotation on thermal instability in couple-stress elastico-viscous fluid, Z. Naturforsch., Vol. 59a, 407-411.

Linden, P.F. (1974). Salt fingers in a steady shear flow, Geophys. Fluid Dynamics, Vol. 6, 1.

Lister, P.E. (1972). On the thermal balance of a midocean ridge, Geophys. J. Roy. Astr. Soc., Vol. 26, 515-535.

McDonnel, J.A.M. (1978). Cosmic Dust, John Wiley and Sons, Toronto, 330.

Nason, P., V. Schumaker, B. Halsalt and J. Schwedes (1969). Formation of a streaming convective disturbance which may occur at one gravity during preparation of samples for zone centrifugation, Biopolymers, Vol. 7, 241-249.

Sharma, R.C., Sunil, Y.D. Sharma and R.S. Chandel (2002). On Couple-stress fluid permeated with suspended particles heated from below, Arch. Mech. (Poland), Vol. 54, 287-298.

Shirtcliffe, T.G.L. (1967). Thermosolutal convection: observation of an overstable mode, Nature (London), Vol. 213, 489-490. 
P. Kumar/ JAFM, Vol. 5, No. 4, pp. 45-52, 2012.

Spiegel, E.A. (1965). Convective instability in a compressible atmosphere, Astrophys. J., Vol. 141, 1068-1090.

Stokes V.K. (1966). Couple-stresses in fluids, Phys. Fluids, Vol. 9, 1709-1715.

Stommel, H. and K.N. Fedorov (1967). Small scale structure in temperature and salinity near Timor and Mindano, Tellus, Vol. 19, 306.

Sunil, Sharma, R.C. and M. Pal (2002). On couple stress fluid heated from below in porous medium in presence of magnetic field and rotation, $J$. Porous Media, Vol. 5, 149-158.

Tabor, H. and R. Matz (1965). Solar pond project, Solar Energy, Vol. 9, 177-182.

Veronis, G. (1965). On finite amplitude instability in thermohaline convection, J. Marine Res., Vol. 23, $1-17$.

Walicki, E. and A. Walicka (1999). Inertia effects in the squeeze film of a couple stress fluid in biological bearing, Appl. Mech. Engng., Vol. 4, 363-373. 